\title{
Tumor Necrosis Factor Receptor Superfamily Member 12A
}

National Cancer Institute

\section{Source}

National Cancer Institute. Tumor Necrosis Factor Receptor Superfamily Member 12A. NCl Thesaurus. Code C117073.

Tumor necrosis factor receptor superfamily member 12A (129 aa, 14 kDa) is encoded by the human TNFRSF12A gene. This protein is involved in both apoptosis and angiogenesis. 